\title{
Responses to Static Visual Images in Macaque Lateral Geniculate Nucleus: Implications for Adaptation, Negative Afterimages, and Visual Fading
}

\author{
Douglas McLelland, Bashir Ahmed, and Wyeth Bair \\ Department of Physiology, Anatomy, and Genetics, University of Oxford, Oxford OX1 3PT, United Kingdom
}

\begin{abstract}
Adaptation to static scenes is a familiar and fundamental aspect of visual perception that causes negative afterimages, fading, and many other visual illusions. To establish a foundation for understanding the neuronal bases of such phenomena and to constrain the contributions of retinal versus cortical processing, we studied the responses of neurons in the dorsal lateral geniculate nucleus during and after the presentation of prolonged static visual stimuli. We found that parvocellular $(\mathrm{P})$ cells (the more numerous and color-sensitive pathway) showed response adaptation with a time constant on the order of tens of seconds and that their response after the removal of a visual stimulus lasting $1 \mathrm{~min}$ was similar in amplitude and time course to the response evoked by the photographic negative stimulus. Magnocellular (M) cells (the faster-conducting and achromatic pathway) had after responses that were substantially weaker than responses evoked by patterned visual stimuli. This difference points to the existence of an adaptive mechanism in the P-pathway that is absent or impaired in the M-pathway and is inconsistent with full adaptation of photoreceptors, which feed both pathways. Cells in both pathways often maintained a substantial tonic response throughout $1 \mathrm{~min}$ stimuli, suggesting that these major feedforward inputs to cortex adapt too slowly to account for visual fading. Our findings suggest that faster-adapting mechanisms in cortex are likely to be required to account for the dynamics of perception during and after the viewing of prolonged static images.
\end{abstract}

\section{Introduction}

A fascination with visual afterimages since the time of Purkinje (1825; Anstis et al., 1978) has produced a wealth of psychophysical studies that probe the nature of visual perception, from retinal mircocircuitry (Wilson, 1997) to neuronal correlates of consciousness (Tsuchiya and Koch, 2006). A perennial debate has been whether the seat of afterimages is retinal or cortical (Parinaud, 1882; Delabarre, 1889; Creed and Harding, 1930; Misiak and Lozito, 1951; Loomis, 1972; Virsu and Laurinen, 1977; Shimojo et al., 2001; Gilroy and Blake, 2005; Tsuchiya and Koch, 2006). A more recent debate has arisen regarding the roles of the functionally segregated parvocellular $(\mathrm{P})$ and magnocellular $(\mathrm{M})$ retinogeniculate pathways (Ingling and Grigsby, 1990; Kelly and Martinez-Uriegas, 1993; Schiller and Dolan, 1994). Despite many attempts to link the perception of afterimages to hierarchical stages and parallel streams of the visual system, we are not aware of any systematic electrophysiological characterizations of the signals discharged by single neurons in retina, lateral geniculate nucleus (LGN), or V1 in response to prolonged presentations of static patterned visual stimuli. The absence of, and need for, studies characterizing slow components of visual adaptation was

Received Jan. 27, 2009; revised April 6, 2009; accepted May 8, 2009.

This work was supported by the Wellcome Trust. B.A. was supported by Biotechnology and Biological Sciences Research Council Grant BBC5049431. We thank Pamela Baker, Mark Baxter, Kristine Krug, Kathy Murphy, and Andrew Parker for assistance with experiments.

Correspondence should be addressed to Douglas McLelland at the above address. E-mail: douglas.mclelland@ dpag.ox.ac.uk.

DOI:10.1523/JNEUROSCI.0467-09.2009

Copyright $\odot 2009$ Society for Neuroscience $\quad$ 0270-6474/09/298996-06\$15.00/0 noted by Shapley and Enroth-Cugell (1984). Such characterizations can offer a new set of constraints for working out functional neuronal circuitry in the visual system. Moreover, understanding how neurons in the primate visual system respond to such stimuli can provide a foundation for interpreting the extensive psychophysical literature on afterimages and fading.

We studied responses in the macaque LGN during and after prolonged visual stimuli presented at moderate luminance on a standard video display [cathode ray tube (CRT)]. Such stimuli are typical of those used in psychophysical and neurophysiological studies and are not associated with significant bleaching of photoreceptors (Shapley and Enroth-Cugell, 1984). We focused on the time scale of seconds to minutes because this covers the ranges associated with visual fading (Tulunay-Keesey, 1982), afterimage formation (Kelly and Martinez-Uriegas, 1993), and luminance adaptation (Baker, 1949; Adelson, 1982; Hayhoe et al., 1992; Yeh et al., 1996; Hood, 1998). Recording in the LGN allowed us to separately study the behavior of the parallel $\mathrm{M}$ - and P-pathways. We hoped to reconcile psychophysical claims that the M-pathway does not mediate afterimages (Ingling and Grigsby, 1990; Kelly and Martinez-Uriegas, 1993) with the demonstration that macaques still report afterimages after P-pathway lesions (Schiller and Dolan, 1994). Furthermore, we measured time constants of adaptation in the LGN to determine whether they match those of visual fading and afterimages in human psychophysics, and we tested the implications of an explicit model of afterimage formation via early retinal circuitry (Wilson, 1997). 


\section{Materials and Methods}

\section{Electrophysiology}

Single-unit responses were recorded extracellularly from the dorsal LGN of two anesthetized, paralyzed macaques (Macaca mulatta). Detailed methods are available in the study by Bair et al. (2002). Animals were anesthetized and paralyzed with sufentanil citrate $(6-30 \mu \mathrm{g} / \mathrm{kg} / \mathrm{h})$ and vecuronium bromide (Norcuron; $0.1 \mathrm{mg} / \mathrm{kg} / \mathrm{h}$ ), in lactated Ringer's solution $(2 \mathrm{ml} / \mathrm{kg} / \mathrm{h})$ containing dextrose $(2.5 \%)$. Anesthesia was supplemented with isoflurane $(0.25 \%)$. Artificial respiration with a moist mixture of $50 \% \mathrm{O}_{2}$ and $50 \%$ room air was maintained with rate adjustments to keep expired $\mathrm{CO}_{2}$ between 32 and $38 \mathrm{mmHg}$. Body temperature was maintained near $37^{\circ} \mathrm{C}$ with a heating pad. EEG and electrocardiogram were monitored to maintain a proper depth of anesthesia. The corneas were protected with gas-permeable hard contact lenses, with additional lenses to optimize neuronal responses to high spatial frequency (SF) stimuli. All procedures conformed to UK Home Office regulations on animal experimentation.

A mechanical microdrive was used to advance quartz-platinum tungsten microelectrodes into the brain (Thomas Recordings). The craniotomy was centered behind the central sulcus and $11 \mathrm{~mm}$ lateral to the midline. Neurons were recorded in the P- and M-layers and had receptive field eccentricities between 10 and $40^{\circ}$. Signals were digitized at 12.5 $\mathrm{kHz}$ using a National Instruments analog-to-digital board, and spikes were discriminated using time-amplitude windows (custom software, C-code) and stored at $1 \mathrm{~ms}$ resolution. Electrolytic lesions were made for histological verification and estimation of LGN layer. After $5 \mathrm{~d}$, animals were given an overdose of sodium pentobarbital ( $65 \mathrm{mg} / \mathrm{kg})$, exsanguinated, and perfused with $4 \%$ paraformaldehyde in saline.

\section{Visual stimuli}

Basic characterization. We mapped each cell by hand using bars and gratings while adjusting the electrode depth in micrometer increments to obtain well isolated action potential waveforms. We next characterized each cell physiologically by presenting on a CRT $(96 \mathrm{~Hz}$; mean luminance, $27 \mathrm{~cd} / \mathrm{m}^{2}$ ) a series of drifting sinusoidal gratings under computer control to generate tuning curves for direction, SF, temporal frequency, and size. We then presented the optimal grating as a static stimulus for $2 \mathrm{~s}$ at eight spatial phases. We defined the preferred phase to be that generating the highest firing rate and the antiphase to be $180^{\circ}$ opposite (see Fig. $1)$.

Static, long duration. To examine adaptation and recovery on a long time scale, we presented $64 \mathrm{~s}$ static stimuli followed by $128 \mathrm{~s}$ of mean gray. Typically, we collected six trials with the antipreferred stimulus and six trials with the preferred stimulus. At the end of most runs, and particularly if an audible change in response occurred, we retested the spatial phase tuning to ensure that eye position had not changed. If a substantial phase shift was detected, the data were discarded.

Static, variable duration. We presented the antipreferred stimulus for varying durations followed by recovery epochs. Six conditions were interleaved, consisting of stimulus durations $1,2,4,8,16$, and $32 \mathrm{~s}$, followed by recovery epochs of $16,2,4,8,8$, and $16 \mathrm{~s}$, respectively. The recovery epochs for 16 and $32 \mathrm{~s}$ stimuli were limited to shorten the overall experiment, and the recovery epoch for $1 \mathrm{~s}$ was matched for comparison with the $32 \mathrm{~s}$ stimulus. These details were designed for testing V1 cells, which typically recovered rapidly and within the epochs provided (McLelland et al., 2007), and are not critical here. Within each block of six conditions, the stimulus was present for $63 \mathrm{~s}$ and absent for $63 \mathrm{~s}$, including $1.5 \mathrm{~s}$ of gray between trials.

Cell classification. We distinguished M- and P-cells based on the presence of contrast gain control (Shapley and Victor, 1978) in M-cells and its absence in P-cells (Benardete and Kaplan, 1999). Classification was based on the temporal kernels (Benardete and Kaplan, 1999; Bair et al., 2002) and was in agreement with our assessment from contrast sensitivity, latency, and laminar location.

\section{Data analysis}

Responses are shown as peristimulus time histograms (PSTHs), convolved with a Gaussian to suppress noise (for SD, see figures) and scaled to show the mean instantaneous firing rate. Where normalized popula- tion PSTHs are presented, we divided the PSTH for each cell by the firing rate in the time window starting at $500 \mathrm{~ms}$ (to avoid the on transient) and ending at $1000 \mathrm{~ms}$. We calculated the amplitude of on- and off-transient responses as the peak firing rate within the first $120 \mathrm{~ms}$ in PSTHs that were smoothed with a Gaussian of SD 4 ms.

We measured the variability of interspike intervals (ISIs) using the CV2 metric (Holt et al., 1996) defined for two consecutive ISIs, $a$ and $b$, as $\mathrm{CV} 2=2|a-b| /(a+b)$. This is simply the difference over the mean of the intervals and is insensitive to slow changes in firing rate because it is local in time.

Exponential functions of the following form were fit to PSTHs, minimizing mean squared error (MSE):

$$
\begin{aligned}
& y(t)=A e^{(-t / \tau)}+B, t \geq 0, \\
& y(t)=0, t<0 .
\end{aligned}
$$

The parameters $A$ and $B$ are the amplitude and offset firing rates, and $\tau$ is the time constant

A model of adaptation was fit to the data for the variable duration stimuli. The model holds that the response, $r(t)$, to the stimulus is proportional to the difference between the stimulus amplitude and the reference adaptation level:

$$
r(t)=s(t)-a(t)
$$

where the adaptation level is given by the following:

$$
a(t)=y(t)^{*} s(t) .
$$

Here, the asterisk indicates convolution, $y(t)$ is an exponential (Eq. 1), and $s(t)$ is the time-varying stimulus contrast, which is zero before the stimulus begins and during recovery epochs and -1 during the antipreferred stimulus. The MSE of the fit was minimized only during the recovery periods, because the firing rates of most cells were suppressed to zero during the antipreferred stimulus, preventing the observation of the temporal evolution of the antipreferred response.

\section{Results}

For each LGN neuron, we identified a preferred static stimulus (see Materials and Methods). The antipreferred stimulus was constructed by shifting the phase of the preferred grating by $180^{\circ}$; thus, it was the photographic negative of the preferred (Fig. 1). The responses to these stimuli can be broken into four major components: the on- and off-transients and the maintained and after responses (Fig. $1 B$ ). The first three components are familiar, as described by Kuffler (1953) and depicted by Enroth-Cugell and Robson (1966) for cat retinal ganglion cells, but the fourth component, the after response, has been essentially overlooked.

To characterize the after response and compare it to the maintained response on a time scale that encompasses the psychophysical fading of stabilized images and negative afterimages (Tulunay-Keesey, 1982; Hayhoe et al., 1992), we presented static stimuli for $64 \mathrm{~s}$, followed by $128 \mathrm{~s}$ of mean gray (Fig. $2 \mathrm{~A}$ ).

The responses of three $\mathrm{P}$-cells to prolonged stimuli are shown in Figure $2 B$. The maintained response of the first cell, which had appeared relatively flat in Figure $1 B$, can be seen to decay substantially at the longer time scale presented here. The response to the antipreferred stimulus was somewhat suppressed during the maintained period but increased rapidly after the stimulus disappeared such that the after response to the antipreferred stimulus (AR) approximated the maintained response to the preferred stimulus (MR). This differs from the case for the $2 \mathrm{~s}$ stimuli (Fig. $1 A, B)$, in which the AR was substantially less than the MR, indicating that the amplitude of the AR depends on the adaptation time. A second example (Fig. $2 B$, middle) shows the same pattern, in which the approximate amplitude and time course of the $\mathrm{MR}$ and AR were similar. The third example P-cell had a more 
complicated time course (Fig. $2 B$, bottom), in which the MR increased over the first $10 \mathrm{~s}$ and then decayed back toward the spontaneous level. This humped time course also appeared in the AR, consistent with the similarity of $\mathrm{MR}$ and $\mathrm{AR}$ in the first two examples.

All P-cells showed behavior consistent with these examples. We normalized the PSTHs for each cell so that 1 represents the firing rate at the start of the MR and 0 represents the baseline rate (see Materials and Methods) and averaged them together (Fig. $2 C)(n=9)$. The resulting population PSTHs reveal several important characteristics of long time-scale adaptation. First, the average MR does not return to baseline, even after $64 \mathrm{~s}$, suggesting that adaptation in the retina and LGN is insufficient for the fading of these stimuli within $64 \mathrm{~s}$. Second, despite the incomplete decay toward baseline during the maintained period, the amplitude of the AR is similar to that of the MR, suggesting that the underlying adaptive mechanism may be near saturation. Third, the dynamics of the after responses approximated those of the maintained responses to the photographic negative stimuli. Thus, the P-pathway delivers a set of signals consistent with a negative afterimage that is similar in time course and amplitude to the original stimulus in terms of the mean instantaneous firing rate.

We tested M-cells with the same stimulus paradigm, with markedly different results. Figure $2 D$ shows the responses of three typical M-cells. Their responses were similar to those of P-cells while the stimuli were present (i.e., MRs were elevated for preferred stimuli, suppressed for antipreferred stimuli, and decayed partially toward a middle value during the $64 \mathrm{~s}$ stimulus presentation). However, when the antipreferred stimulus disappeared at $t=$ $64 \mathrm{~s}$, the ARs were substantially less than the MRs had been to the preferred stimuli and thus less than would have been expected for P-cells. This difference in behavior is summarized by the population average PSTHs (Fig. 2 E), which show that M-cells provide the cortex with signals consistent with a negative afterimage but substantially weaker than that associated with the actual negative stimulus and similarly weaker than that transmitted by P-cells (Fig. 2, compare $C, E$ ).

We quantified the time courses of MRs and ARs by fitting exponential functions (Eq. 1; see Materials and Methods) to the PSTHs. When fitting, we omitted the first 10 s period, which often contained marked deviations from exponential decay (e.g.,
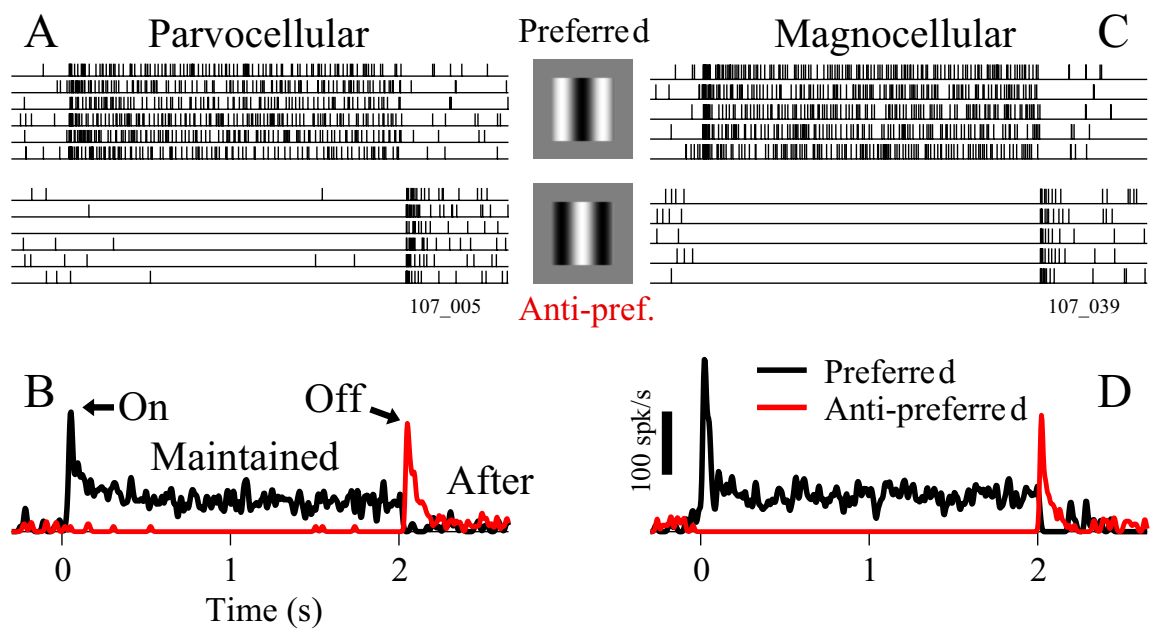

Figure 1. Preferred and antipreferred static stimuli. $\boldsymbol{A}$, P-cell spike train rasters ( 6 trials) in response to a preferred (top) and antipreferred (bottom) stimulus. B, PSTHs, convolved with a Gaussian (SD, 4 ms) for preferred (black) and antipreferred (red) responses. Four response components are indicated. $C, D$, Similar results for an M-cell.
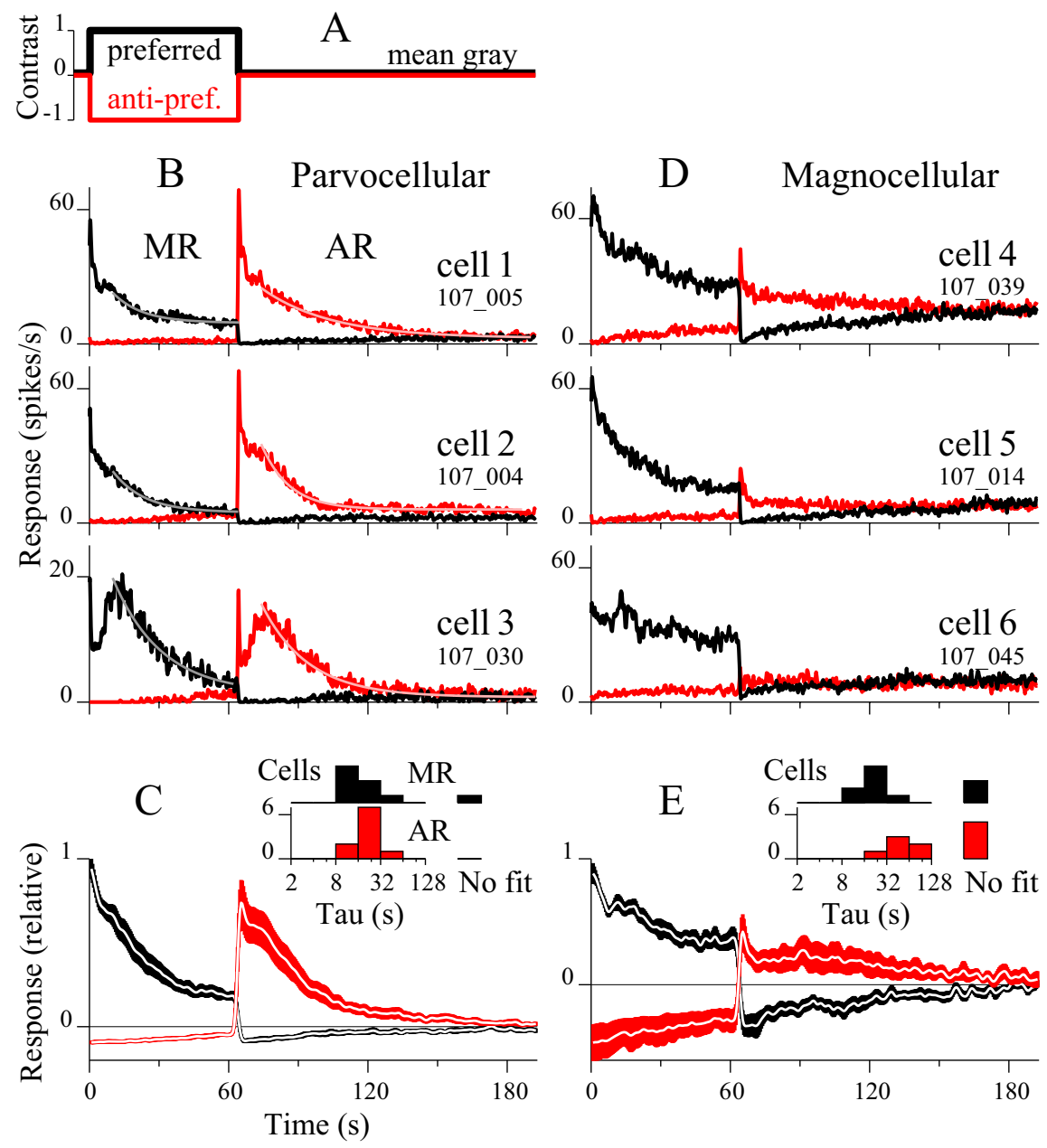

Figure 2. Comparison of MR and AR for $P$ - and M-cells. $A$, Stimulus timing diagram. Stimulus contrast is plotted versus time. anti-pref., Antipreferred. $\boldsymbol{B}$, PSTHs for three P-cells. The first cell is that of Figure 1. The MR and AR are shown for the preferred (black line) and antipreferred (red line) stimulus. PSTHs are averages of six trials, convolved with a Gaussian (SD, $300 \mathrm{~ms}$; the difference in smoothing between here and Fig. 1 results in the apparent change in peak firing rates). Smooth, light traces are exponential fits. C, Population PSTHs for P-cells $\left[n=9\right.$ (80FF cells, $10 \mathrm{~N}$ cell); see Materials and Methods]. Inset, $\tau_{\mathrm{MR}}$ and $\tau_{\mathrm{AR}}$. Cells counted as "No fit" had responses that were too flat or too noisy. Mean values are $17 \mathrm{~s}(\mathrm{SD}, 8 ; n=9)$ and $21 \mathrm{~s}(\mathrm{SD}, 9 ; n=10)$ for MR and AR, respectively. $\boldsymbol{D}$, Like $\boldsymbol{B}$, but for M-cells. $\boldsymbol{E}$, Like $\boldsymbol{C}$, but for M-cells $[n=11$ (70FF cells, $40 \mathrm{~N}$ cells)]. Inset, Means are $22 \mathrm{~s}$ $(S D, 8 ; n=8)$ and 49 s (SD, 27; $n=6)$ for MR and $A R$, respectively. 


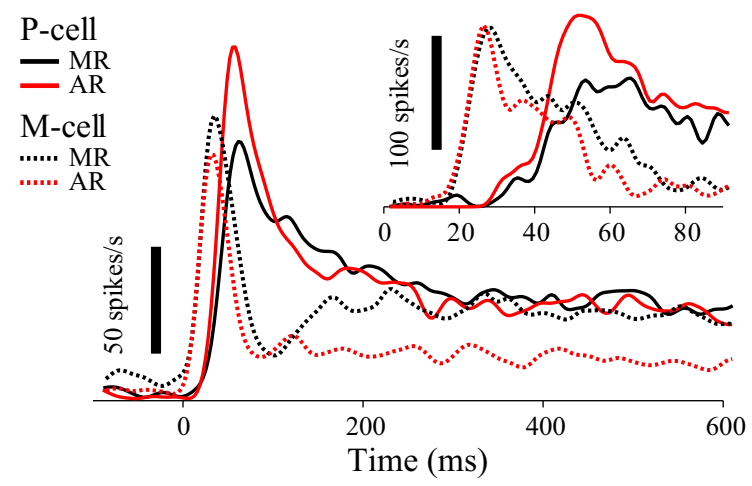

Figure 3. High-resolution comparison of early responses. PSTHs averaged over nine P-cells (solid curves) and nine M-cells (dotted curves). Time 0 is stimulus onset for on/maintained responses (black lines) and stimulus offset for off/after responses (red lines). Curves are smoothed by Gaussian convolution (SD, $10 \mathrm{~ms}$ ). Inset, Same data at higher (SD, 2 ms) resolution.

cell 3 in Fig. $2 B$ ). The distributions of time constants $\tau_{\mathrm{MR}}$ and $\tau_{\mathrm{AR}}$ across cells are shown in Figure 2, $C$ and $E$ (insets). For P-cells, there was no significant difference between $\tau_{\mathrm{MR}}$ and $\tau_{\mathrm{AR}}(t$ test, $p=0.23 ; \mathrm{n} 1=9 ; \mathrm{n} 2=10)$. For M-cells, $\tau_{\mathrm{AR}}$ was significantly larger than $\tau_{\mathrm{MR}}(t$ test, $p=0.02 ; \mathrm{n} 1=8 ; \mathrm{n} 2=6)$; however, we believe this is related to low AR amplitudes in some M-cells (e.g., cell 6 in Fig. 2D). In fact, many ARs were basically flat and could not be reliably fit, as indicated by the histogram bars marked "No fit" in Figure $2 E$ (inset). $\tau_{\mathrm{MR}}$ was not significantly different between P- and M-cells $(p=0.17 ; \mathrm{n} 1=9 ; \mathrm{n} 2=8)$, but $\tau_{\mathrm{AR}}$ was larger for M-cells $(p=0.01 ; \mathrm{n} 1=10 ; \mathrm{n} 2=6)$. Overall, $\tau$ values were, on average, $\sim 20 \mathrm{~s}$ (Fig. 2) but somewhat longer for M-cell ARs ( $\sim 50 \mathrm{~s})$. Thus, a major component of adaptation in the MRs and ARs operates on the order of tens of seconds.

Given the similarity of the P-cell AR and MR in terms of mean firing rate, we wondered whether they could be distinguished based on response variability. We found no difference in spike count variance or in the ISI variability metric, CV2 (Holt et al., 1996) (see Materials and Methods). In particular, the spike count variance was not significantly different when comparing the first $20 \mathrm{~s}$ of the MR to the first $20 \mathrm{~s}$ of the $\operatorname{AR}(p=0.71$, paired $t$ test). The CV2 metric varied considerably across cells (0.56-1.1), was highly correlated between MR and AR $(r=0.91 ; p<0.001 ; n=$ $9)$, and also did not differ significantly when comparing MR with AR ( $p=0.74$, paired $t$ test). We performed similar tests for 10 and $60 \mathrm{~s}$ epochs and found similar results. For M-cells, we found no difference in spike count variance and a slight increase in ISI variability for the AR, but the latter was expected, being correlated with the decrease in firing rate during the AR compared with the MR.

Having observed that the M-cell AR was substantially weaker than the MR, we tested whether the off-transient (Fig. 1) was likewise weaker than the on-transient. We found no significant difference between the amplitude (see Materials and Methods) of on- and off-transient responses for M-cells ( $p=0.54 ; n=11)$. This also held for P-cells (paired $t$ test, $p=0.35 ; n=9$ ). Thus, the difference between the MR and AR for M-cells (Fig. 2E) was observed only after the transient responses. A high-resolution comparison of the population PSTHs for the relevant part of the response is shown in Figure 3, where off/after responses are overlaid with on/maintained responses. As expected, M-cell on- and off-transients occurred $\sim 20 \mathrm{~ms}$ earlier than P-cell responses (Fig. 3, inset) (Maunsell et al., 1999), and M-cell responses were more transient in the first $\sim 100 \mathrm{~ms}$, agreeing with the classical distinc- tion made by Schiller and Malpeli (1978). Shortly after $100 \mathrm{ms,}$ there is a clear difference between the M-cell AR and MR that is not present for $\mathrm{P}$-cells. The M-cell AR is significantly less than the MR in the epoch from 200 to $600 \mathrm{~ms}$ (paired $t$ test, $p=0.01 ; n=$ 9). In summary, the M-cell difference arises early, although it is not immediately evident in the on-transient, perhaps as a result of saturation of the high-amplitude transient signals.

The results above suggest that the dynamics of P-cell MRs and ARs are similar and could be unified with a simple adaptive model (see Materials and Methods) in which the response depends not directly on stimulus contrast but on the difference (Eq. 2 ) between the true contrast and a reference signal that is constantly adapting to match the stimulus (Eq. 3). We tested whether this form of adaptation could approximate the ARs of P-cells on a single-trial basis in response to a richer stimulus in which antipreferred gratings were presented at variable times and with variable durations. A $400 \mathrm{~s}$ response epoch from example cell 1 (Fig. $2 B$ ) is shown in Figure $4 B$ along with the AR predicted by the model (black line). The fit captures several prominent features of the data. The ARs after the first few stimuli have lower amplitudes (e.g., arrow 1) than later ones (arrow 3), showing that adaptation builds up across stimuli. The AR decays progressively across stimuli when the antipreferred stimuli are infrequent and brief (arrow 4). Finally, the off-transient (arrow 2), which is not modeled here, is strong from the beginning and does not change in proportion to the AR.

The time constants for these fits (Fig. 4C) were longer, on average, than those of Figure 2. This reflects the fact that fitted epochs are shorter and the fits here begin at the start of each AR, whereas those in Figure 2 started 10 s later to avoid the early part of the response, which in some cells was not well modeled by decaying exponentials (being flat or leading to a hump). Nevertheless, results from both experiments demonstrate that time constants of adaptation to static stimuli are on the order of tens of seconds. We did not fit M-cells because they had weaker and flatter ARs, on average.

\section{Discussion}

We have identified a component of adaptation to static visual patterns that operates on a time scale of $10-100 \mathrm{~s}$ and is present in the outputs of both P- and M-cells in the LGN. Both pathways showed similar adaptation during prolonged static stimuli. However, whereas ARs in the M-pathway were consistently weaker than the driven response to the photographic negative, ARs in the P-pathway approximated the driven response. Under the assumption of rate coding, as in the fifth dogma of Barlow (1972), signals in the P-pathway that adapt completely could support a perceptual equivalence between real images and afterimages. This, however, is undermined by the M-pathway, which maintains a distinction by virtue of its incomplete adaptation. Nevertheless, both pathways carry ARs that are significant and decay slowly over many tens of seconds.

These results help to resolve some conflict between past studies. The idea that the M-pathway is completely transient, having only brief on- and off-responses, is inconsistent with our findings, yet this was put forward by Ingling and Grigsby (1990) in a psychophysical study concluding that afterimages are solely $\mathrm{P}$ driven. Their conclusion was echoed by Kelly and MartinezUriegas (1993), who found the intriguing result that afterimages from both chromatic and achromatic gratings elevated the psychophysical threshold for detecting chromatic gratings, but neither raised the threshold for achromatic gratings. Schiller and Dolan (1994) tested this P-only afterimage hypothesis by lesion- 
ing the LGN P-layers in macaque and found that subjects continued to reliably report visual afterimages. They concluded that the M-pathway must be capable of supporting afterimages. Our results support this conclusion, as both $\mathrm{M}$ - and P-pathways showed clear ARs, but also offer a basis for explaining why achromatic detection thresholds are insensitive to afterimages, as many $\mathrm{M}$-cells showed weak ARs. Thus, it is possible that such M-cells retain enough of their dynamic range to support low detection thresholds even as others carry signals strong enough to spark awareness of an afterimage, but a systematic study of sensitivity during the after response would be required to test this idea.

Our results also shed light on plausible retinal circuits for local luminance adaptation. Wilson (1997) argued that a slow subtractive process of light adaptation (Hayhoe et al., 1992) that underlies afterimage formation is mediated by the gain of the negative feedback from horizontal cells onto cones. However, this model predicts similar ARs for parasol and midget gan-

glion cells because the adaptive mechanism operates early in elements that are common to both pathways (cones and H1 horizontal cells). Our data show substantial differences in $\mathrm{M}$ and $\mathrm{P}$ ARs, suggesting that the relevant mechanism lies in cells that are distinct in these pathways: bipolar, amacrine or ganglion cells (Dacey, 2000). We hypothesize the existence of slow, subtractive adaptation (Adelson, 1982) like that in our model (see Materials and Methods) in the midget pathway that is absent or weaker in the parasol pathway. An alternative hypothesis, that the adaptation occurs at the photoreceptors and is subsequently suppressed in the parasol pathway, is less attractive because it would require the M-pathway to somehow selectively suppress ARs but not MRs. It seems simpler to assume that strong ARs never develop in the first place in the M-pathway.

We think it is unlikely that our results are closely linked to the growing body of literature on contrast gain control (Shapley and Enroth-Cugell, 1984; Solomon et al., 2006), which operates nearly three orders of magnitude faster and involves adaptation to temporal variation around a mean rather than to a static luminance pattern. Perhaps a more relevant study is that of Yeh et al. (1996), who probed the sensitivity of macaque retinal responses in the $30 \mathrm{~s}$ after step changes in full-field (unpatterned) luminance. In contrast with our results, for achromatic stimuli they reported a rapid $(\sim 2-3 \mathrm{~s})$ return to baseline response levels and low overall responses. This is consistent with their full-field stimuli engaging center-surround antagonism, thereby minimizing the driven response. Our patterned stimuli engaged the center and surround synergistically, causing strong elevations (or depressions) in firing rate, not present for their stimuli. Notably, for chromatic stimuli, which should not engage center-surround antagonism, they found strong responses and longer time courses of recovery that are consistent with our results.

The time scale of adaptation characterized here bears comparison to a large body of psychophysical literature on light adaptation, visual fading, and afterimages. Baker's (1949) study of light adaptation yields $\tau$ values of, on average, $25 \mathrm{~s}$ and complete adaptation within $3 \mathrm{~min}$. Anstis et al. (1978) reported $\tau=25-54 \mathrm{~s}$ for the decay of contrast afterimages and found that $18 \mathrm{~s}$ stimuli were too short to build full-strength afterimages. These reports, for example, approximately match our data. However, one of the most thorough investigations of the time course of afterimages (Kelly and Martinez-Uriegas, 1993) found that chromatic and achromatic afterimages developed and decayed with similar exponential time constants, $4-8 \mathrm{~s}$, consistent with the 5-9 s reported previously for achromatic afterimages (Virsu and Laurinen, 1977). These numbers are substantially shorter than our values for the mean time constants of LGN ARs. Hayhoe et al. (1992) identified a subtractive process of light adaptation that asymptotes within $10-15 \mathrm{~s}$ and is believed to be consistent with the time course of visual fading, which occurs on the order of a few seconds (King-Smith et al., 1977; Tulunay-Keesey, 1982). These results, not to mention common experience with a variety of popular visual illusions, suggest that visual fading and afterimages typically operate on a substantially faster time scale than that required for LGN responses to return to their baseline levels.

Thus, although classical studies have provided compelling explanations of visual psychophysics on the basis of retinal adaptation (Barlow and Levick, 1969; Shapley and Enroth-Cugell, 1984), the slowly adapting signals and long-lasting maintained responses observed in the LGN suggest that adaptation in the cortex, not the retina, will be required to account for fading and afterimages. Preliminary data from V1 suggests that most cortical cells adapt back to their spontaneous firing rates within a few seconds (McLelland et al., 2007).

\section{References}

Adelson EH (1982) Saturation and adaptation in the rod system. Vision Res 22:1299-1312.

Anstis S, Rogers B, Henry J (1978) Interactions between simultaneous contrast and coloured afterimages. Vision Res 18:899-911.

Bair W, Cavanaugh JR, Smith MA, Movshon JA (2002) The timing of response onset and offset in macaque visual neurons. J Neurosci 22:3189-3205.

Baker HD (1949) The course of foveal light adaptation measured by the threshold intensity increment. J Opt Soc Am 39:172-179. 
Barlow HB (1972) Single units and sensation: a neuron doctrine for perceptual psychology? Perception 1:371-394.

Barlow HB, Levick WR (1969) Three factors limiting the reliable detection of light by retinal ganglion cells of the cat. J Physiol 200:1-24.

Benardete EA, Kaplan E (1999) The dynamics of primate M retinal ganglion cells. Vis Neurosci 16:355-368.

Creed RS, Harding RD (1930) Latency of after-images and interaction between the two retino-cerebral apparatuses in man. J Physiol 69:423-441.

Dacey D (2000) Parallel pathways for spectral coding in primate retina. Annu Rev Neurosci 23:743-775.

Delabarre EB (1889) On the seat of optical after-images. Am J Psychol 2:326-328.

Enroth-Cugell C, Robson JG (1966) The contrast sensitivity of retinal ganglion cells of the cat. J Physiol 187:517-552.

Gilroy LA, Blake R (2005) The interaction between binocular rivalry and negative afterimages. Curr Biol 15:1740-1744.

Hayhoe MM, Levin ME, Koshel RJ (1992) Subtractive processes in light adaptation. Vision Res 32:323-333.

Holt GR, Softky WR, Koch C, Douglas RJ (1996) Comparison of discharge variability in vitro and in vivo in cat visual cortex neurons. J Neurophysiol 75:1806-1814.

Hood DC (1998) Lower-level visual processing and models of light adaptation. Annu Rev Psychol 49:503-535.

Ingling CR Jr, Grigsby SS (1990) Perceptual correlates of magnocellular and parvocellular channels: seeing form and depth in afterimages. Vision Res 30:823-828.

Kelly DH, Martinez-Uriegas E (1993) Measurement of chromatic and achromatic afterimages. J Opt Soc Am A 10:29-37.

King-Smith PE, Riggs LA, Moore RK, Butler TW (1977) Temporal properties of the human visual nervous system. Vision Res 17:1101-1106.

Kuffler SW (1953) Discharge patterns and functional organization of mammalian retina. J Neurophysiol 16:37-68.

Loomis JM (1972) The photopigment bleaching hypothesis of complementary after-images: a psychophysical test. Vision Res 12:1587-1594.

Maunsell JHR, Ghose GM, Assad JA, McAdams CJ, Boudreau CE, Noerager
BD (1999) Visual response latencies of magnocellular and parvocellular LGN neurons in macaque monkeys. Vis Neurosci 16:1-14.

McLelland D, Ahmed B, Bair W (2007) Neuronal correlates of visual afterimages in macaque LGN and visual cortex. Soc Neurosci Abstr 33:395.21.

Misiak H, Lozito CC (1951) Latency and duration of monocular and binocular after-images. J Exp Psychol 42:247-249.

Parinaud H (1882) Gaz Hôpitaux 55:459-460.

Purkinje JE (1825) Beobachtungen und Versuche zur Physiologie der Sinne, Vol 2, p 107. Berlin: G. Reimer.

Schiller PH, Dolan RP (1994) Visual aftereffects and the consequences of visual system lesions on their perception in the rhesus monkey. Vis Neurosci 11:643-665.

Schiller PH, Malpeli JG (1978) Functional specificity of lateral geniculate nucleus laminae of the rhesus monkey. J Neurophysiol 41:788-797.

Shapley R, Enroth-Cugell C (1984) Visual adaptation and retinal gain controls. In: Progress in retinal research, Vol 3 (Osborn N, Chader G, eds), pp 263-343. Oxford: Pergamon.

Shapley RM, Victor JD (1978) The effect of contrast on the transfer properties of cat retinal ganglion cells. J Physiol 285:275-298.

Shimojo S, Kamitani Y, Nishida S (2001) Afterimage of perceptually filled-in surface. Science 293:1677-1680.

Solomon SG, Lee BB, Sun H (2006) Suppressive surrounds and contrast gain in magnocellular-pathway retinal ganglion cells of macaque. J Neurosci 26:8715-8726.

Tsuchiya N, Koch C (2006) Continuous flash suppression reduces negative afterimages. Nat Neurosci 8:1096-1101.

Tulunay-Keesey U (1982) Fading of stabilized retinal images. J Opt Soc Am 72:440-447.

Virsu V, Laurinen P (1977) Long-lasting afterimages caused by neural adaptation. Vision Res 17:853-860.

Wilson HR (1997) A neural model of foveal light adaptation and afterimage formation. Vis Neurosci 14:403-423.

Yeh T, Lee BB, Kremers J (1996) The time course of adaptation in macaque retinal ganglion cells. Vision Res 36:913-931. 